\title{
Whole-genome sequencing of an advanced case of small-cell gallbladder neuroendocrine carcinoma
}

Maolan $\mathrm{Li}^{1,2,5}$, Fatao Liu ${ }^{1,2,5}$, Yijian Zhang ${ }^{1,2,5}$, Xiangsong $\mathrm{Wu}^{1,2}$, Wenguang $\mathrm{Wu}^{1,2}, \mathrm{Xu}-\mathrm{An}$

Wang $^{1,2}$, Shuai Zhao ${ }^{1,2}$, Shibo Liu ${ }^{1,2}$, Haibin Liang ${ }^{1,2}$, Fei Zhang ${ }^{1,2}$, Yuan Gao ${ }^{1,2}$, Shanshan Xiang ${ }^{1,2}$, Huaifeng $\mathrm{Li}^{1,2}$, Wei $\mathrm{Lu}^{1,2}$, Hao Weng ${ }^{1,2}$, Jiasheng $\mathrm{Mu}^{1,2}$, Yijun Shu ${ }^{1,2}$, Runfa Bao $^{1,2}$, Lin Jiang ${ }^{1,2}$, Yunping $\mathrm{Hu}^{1,2}$, Wei Gong ${ }^{1,2}$, Yun Zhang ${ }^{3}$, Tieliang $\mathrm{Ma}^{3}$, Kai Zhang ${ }^{3,6}$, Yun $\mathrm{Liu}^{4,6}$, Yingbin $\mathrm{Liu}^{1,2,6}$

1Department of General Surgery, Xinhua Hospital Affiliated to Shanghai Jiao Tong University School of Medicine, Shanghai, 200092, China.

2Institute of Biliary Tract Disease, Shanghai Jiao Tong University School of Medicine, Shanghai, 200092, China.

3Dapartment of Hepatobiliary and Laparoscopic Surgrey, the Affiliated Yixing Hospital of Jiangsu University, Yixing, 214200, China.

4Institutes of Biomedical Sciences, Fudan University, Shanghai, 200433, China.

5These authors contributed equally to this work.

6These authors jointly directed this work. Correspondence should be addressed to Y.B.L. (liuybphd@126.com), Y.L. (superliuyun@ fudan.edu.cn) or K.Z. (zk_104@163.com ). 


\begin{abstract}
The majority of gallbladder cancer cases are discovered at later stages, which frequently leads to poor prognoses. Small-cell gallbladder neuroendocrine carcinoma (GB-SCNEC) is a relatively rare histological type of gallbladder cancer, and its survival rate is exceptionally low because of its greater malignant potential. In addition, the genomic landscape of GB-SCNEC is rarely considered in treatment decisions. We performed whole-genome sequencing on an advanced case of GB-SCNEC. By analyzing the whole-genome sequencing data of the primary cancer tissue $(76.29 \mathrm{X}$ coverage), lymphatic metastatic cancer tissue $(73.92 \mathrm{X}$ coverage) and matched non-cancerous tissue (35.73X coverage), we identified approximately 900 high-quality somatic single nucleotide variants (SNVs), 109 of which were shared by both the primary and metastatic tumor tissues. Somatic non-synonymous coding variations with damaging impact in HMCN1 and CDH10 were observed in both the primary and metastatic tissue specimens. A pathway analysis of the genes mapped to the SNVs revealed gene enrichment associated with axon guidance, ERBB signaling, sulfur metabolism and calcium signaling. Furthermore, we identified 20 chromosomal rearrangements that included 11 deletions, 4 tandem duplications and 5 inversions that mapped to known genes. Two gene fusions, NCAM2-SGCZ and BTG3-CCDC40 were also discovered and validated by Sanger sequencing. Additionally, we identified genome-wide copy number variations and microsatellite instability. In this study, we identified novel biological markers of GB-SCNEC that may serve as valuable prognostic factors or indicators of treatment response in patients with GB-SCNEC with lymphatic metastasis.
\end{abstract}




\section{Introduction}

Gallbladder cancer is the most common aggressive tumor of the biliary tract and accounts for $80-95 \%$ of these types of cancers worldwide(Lai and Lau 2008). Because symptoms are frequently indiscernible in the early stages, patients are often diagnosed at more advanced stages of the disease. As a result, the median 5-year survival rate associated with gallbladder cancer remains low(Zhu et al. 2010). Adenocarcinomas account for most gallbladder malignancies (90\%), which include the less common squamous, adenosquamous, and neuroendocrine carcinoma (NEC) histologic subtypes. NEC commonly arises in the gastrointestinal tract and the bronchi but is rarely observed in the gallbladder(Yao et al. 2008). Small-cell neuroendocrine carcinoma (GB-SCNEC) of the gallbladder is even less common and has an incidence of approximately $0.5 \%$ of all gallbladder cancers(Henson et al. 1992) and $0.2 \%$ of all gastrointestinal carcinomas according to Surveillance, Epidemiology and End Results (SEER) data(Jun et al. 2006). The symptomatology of GB-SCNEC is nonspecific, and the diagnosis of GB-SCNEC is often based on the detection of cholecystolithiasis or polyps by cholecystectomy. Women and patients with cholelithiasis are considered high-risk populations for GB-SCNEC, and approximately $75 \%$ of patients from these populations present with concomitant hepatic, pulmonary, peritoneal and lymph node metastases. Because of the rarity of this disease, standards of care for treatment have not been clearly defined, and effective treatment options are currently limited to complete surgical resection with negative margins(El Fattach et al. 2015). Although GB-SCNEC cases represent a relatively small proportion of the total gallbladder cancer cases, the survival rate for neuroendocrine carcinomas is much less that the overall rate for gallbladder cancer because of its malignant potential and late-stage diagnosis. Thus, novel insights into the molecular and genetic aberrations specific to GB-SCNEC might facilitate the development of more efficient methods for the prevention, early diagnosis and curative treatment of this disease.

Gene mutations associated with gallbladder cancers are typically somatic in nature rather than inherited(2015). Mutations and overexpression of the p53 gene are the most common genetic changes observed in human cancers(Itoi et al. 1997), and p53 mutations are observed in up to $70 \%$ of the gallbladder cancer cases evaluated in various studies(Saetta 2006). In a previous study, we observed a significant frequency of non-silent p53 mutations in gallbladder cancer (47.1\%)(Li et al. 2014). K-ras is another well-studied gene implicated in gallbladder cancer. Recent investigations have shown that K-ras mutation rates in various cancers range from 10 to $67 \%$ (Saetta 2006). We previously reported that a substantial proportion of K-ras mutations in 
gallbladder cancer are non-silent (7.8\%), which is similar to p53(Li et al. 2014). The ERBB signaling pathway has the most well-established association with gallbladder carcinogenesis. Mutations in the components of the ERBB signaling pathway, including EGFR, ERBB2, ERBB3 and ERBB4, are the most frequently observed mutations in gallbladder cancer and affect approximately $37 \%$ of all gallbladder tumors( $\mathrm{Li}$ et al. 2014). In a recent study, Miyahara $\mathrm{N}$ demonstrated that Muc4 activation of ERBB2 signaling contributed to gallbladder carcinogenesis(Miyahara et al. 2014). Microsatellite instability (MSI) is another notable genetic factor associated with gallbladder cancer, and the frequency of MSI ranges from $3 \%$ to $35 \%$ (Saetta 2006).

Whole-genome sequencing (WGS) is considered a more powerful method for identifying putative disease-causing mutations in exonic regions compared with whole-exome sequencing (WES)(Belkadi et al. 2015); however, WGS studies in gallbladder cancer and metastatic gallbladder cancer have not been reported. The present study is the first to report the application of WGS technology to study genome-wide single nucleotide variants (SNVs), structural variations, copy number variations $(\mathrm{CNVs})$ and microsatellite stability in a patient with GB-SCNEC. We also evaluated genomic differences between primary and metastatic GB-SCNEC tissues. Together, our findings might provide insights into genome-wide aberrations that mediate gallbladder carcinogenesis and metastasis.

\section{Materials and Methods}

\section{Clinicopathological characteristics of the patient}

A 56-year-old man was hospitalized with a 1-month history of pain in the right hypochodrium. The physical examination revealed no abnormalities. On admission, tumor marker levels were within the normal range. An abdomen CT with and without contrast revealed markedly abnormal gallbladder morphology with an enlargement at the neck region measuring $4.7 \times 4.5 \mathrm{~cm}$. The porta hepatis lymph nodes were abnormally enlarged, and stones were not visible in the gallbladder mass (Figure 1A, 1B). No neoadjuvant or adjuvant chemotherapy was administered prior to surgery. The surgical procedure (which included cholecystectomy as well as extensive surgical resections, including segment 4 of the liver and regional lymph node clearances) was performed in July 2014. After the surgical resections, histopathological analyses revealed that the tumor was composed of monomorphic cells containing small round nuclei and eosinophilic cytoplasm. The cells were organized in small nodular, trabecular or acinar structures surrounded by highly vascularized stroma with a high mitotic index (Figure 1C, 1D). 
Immunohistochemistry assays revealed that the cells in both the tumor and the resected lymph nodes were negative for chromogranin A but positive for synaptophysin, NSE and Ki 67 (80\% of cells) (Figure 1E-1L). These findings were consistent with a diagnosis of GB-SCNEC with lymph node involvement. All of the surgical margins were negative, and a pathological R0 resection was achieved. The patient later received systemic chemotherapy with VP-16 and cisplatin and died 17 months after the operation.

\section{Whole-genome sequencing}

Genomic DNA was extracted from the tissue specimens using the QIAamp DNA kit (Qiagen). The libraries were prepared using the protocols provided by Illumina. Briefly, $1 \mu \mathrm{g}$ of DNA was sheared into small fragments (approximately 200-300 bp) using a Covaris S220 system. DNA fragments were end repaired and 3' polyadenylated. Adaptors with barcode sequences were ligated to both ends of the DNA fragments. E-Gel was used to select the target-size DNA fragments. Then, the tissues underwent 10 cycles of PCR amplification, and the resulting PCR products were purified. Validated DNA libraries were sequenced using the Illumina Sequencing System (HiSeq Series).

\section{Whole-genome sequencing data processing and mutation identification}

Read pairs (FASTQ data) generated from the sequencing system were trimmed and filtered using Trimmomatic(Bolger et al. 2014). The resulting reads were mapped to the human reference genome hg19 downloaded from the UCSC Genome Browser using BWA-MEM (http://arxiv.org/abs/1303.3997)) in the SpeedSeq framework(Chiang et al. 2015). Duplicate marking, position sorting and BAM file indexing were also conducted during the SpeedSeq align procedure using SAMBLASTER(Faust and Hall 2014) and Sambamba (https://github.com/lomereiter/sambamba). The sorted and indexed BAM files were directly loaded into the SpeedSeq somatic module. SpeedSeq analysis using FreeBayes (http://arxiv.org/abs/1207.3907) was performed on the tumor/normal pair of BAM files to achieve somatic SNVs and small INDELs. For the indexed BAM files, we also conducted INDEL realignment and base quality score recalibration using the Genome Analysis Toolkit (GATK)(McKenna et al. 2010), and then the MuTect algorithm(Cibulskis et al. 2013) was applied to identify somatic SNVs. BEDtools was used to filter the somatic SNVs identified by both SpeedSeq and Mutect. Finally, we used the Ensembl Variant Effect Predictor (VEP)(McLaren et al. 2010) to annotate the merged vcf file. 


\section{Pathway analysis}

We performed a pathway analysis of the genes that mapped to the somatic SNVs in the tumor samples using DAVID (https://david.ncifcrf.gov/). Genes listed on the DAVID web site and all known human genes were used as the background. A KEGG database analysis was used to evaluate the pathway enrichment.

\section{Structural variation analysis}

LUMPY was run in the SpeedSeq sv module to identify somatic structural variations(Layer et al. 2014), and the vcf files generated by SpeedSeq were annotated using the VEP algorithm. We predicted the somatic structural variations in the primary and lymphatic metastatic GB-SCNECs using DELLY. We identified large somatic deletions, duplications and inversions detected by both LUMPY and DELLY. With respect to the genomic translocational structural variants, we focused on putative gene fusions. Somatic genomic translocational events were generated and filtered by DELLY. OncoFuse(Shugay et al. 2013) was used to identify the putative activating gene fusions.

\section{Copy number variation analysis}

Saas-CNV(Zhang and Hao 2015) was used to study somatic copy number variations in the primary and metastatic GB-SCNEC tissues. The GATK variant analysis pipeline was used to process the data generated from the sequencing system as described above. High-quality SNVs and small INDELs called by GATK were saved in variant call format (VCF) files, and the files were loaded into the Saas-CNV algorithm. The read depth and alternative allele frequency at each site were determined and used to identify somatic copy number alterations (SCNAs).

\section{Microsatellite stability analysis}

We used the vcf2MSAT (http://sourceforge.net/projects/vcf2msat/) script to retrieve the short tandem repeat (STR) data from the vcf files generated from the SpeedSeq somatic module. Information regarding the microsatellite repeat regions identified as INDELs was saved in the vcf files. The data obtained from identifying the genomic location and somatic changes in repeat number were used for the follow-up analyses.

\section{Results}




\section{Whole-genome sequencing (WGS) and identification of SNVs}

We performed WGS of the primary cancer, lymphatic metastatic cancer and matched non-cancerous tissue specimens. The sequencing data were mapped to the human reference genome hg19 with a mean sequencing depth of 61.98X (range 35.73-76.29X) and a mean coverage of $98.7 \%$. Additional information regarding the WGS data is summarized in

\section{Supplementary Table 1.}

Using the SpeedSeq framework and GATK-MUTECT with the non-cancerous gallbladder tissue as the normal reference, we identified 295 and 712 high-quality somatic SNVs in the primary and lymphatic metastatic tumor tissues, respectively (Figure 2, 3A, 3B). One hundred nine somatic SNVs were shared by the primary and metastatic tumors. Additional information regarding the 109 somatic SNVs is listed in Supplementary Table 2. The majority of the identified SNVs were located in intronic and intergenic regions (Figure 2). A pathway analysis of the genes mapped to the somatic SNVs revealed a significant enrichment in genes associated with axon guidance, ERBB signaling, sulfur metabolism and calcium signaling (Figure 4A). Notably, we identified somatic SNVs in 7 genes (PAK7, HRAS, ERBB4, SOS1, MTOR, NRG1 and ABL1) in the ERBB signaling pathway, which is consistent with the results of our previous study( Li et al. 2014).

Next, we investigated the impact of the genetic variations on amino acid changes. Somatic non-synonymous coding variations in $\mathrm{HMCN} 1$ and $\mathrm{CDH} 10$ were observed in the both the primary and metastatic tissue specimens (Table 1). In addition, somatic non-synonymous coding variations in MAST2, C6orf118, UBR5, OR51F1, SLC22A8, CSPG4 and BAIAP2L2 were observed in the metastatic GB-SCNEC tissue. The SNVs associated with amino acid variations were further validated using conventional Sanger sequencing.

We further evaluated the impact of the SNVs in HMCN1 and CDH10 on the structure and function of proteins using Polyphen2. We found that the amino acid changes in the proteins encoded by the HMCN1 (E171K) and CDH10 (R4623W) variants were predicted to disrupt the structure and function of proteins (score $=0.996$ and 0.880, respectively) (Figure 4B). The two variants were also clearly validated by Sanger sequencing (Figure 4C).

\section{Analysis of structural variations}

Using LUMPY and Delly, we identified more than 50 somatic genomic rearrangements in the primary and lymphatic metastatic GB-SCNECs tissue samples, which included 11 deletions, 4 tandem duplications and 5 inversions mapped to known genes. Additional information 
regarding the genes that mapped to structural variations is summarized in Table 2 , and the genomic locations of the structural variations are indicated in Figure 3A and Figure 3B. Notably, 2 tandem duplications that encompassed the CLCNKA, CLCNKB, FAM131C, C1DP2 and C1DP3 genes were observed in both the primary and lymphatic metastatic GB-SCNEC samples.

We also considered the tanslocational events generated by DELLY. The high-quality somatic translocational structure variants were filtered. In primary and lymphatic metastatic tumor tissues, we identified 35 and 41 translocations respectively, 10 of which were shared by the primary and metastatic tumors. The detailed information regarding the translocations is summarized in Supplementary Table 3. A total of 6 gene fusions were identified , 2 of which , including NCAM2-SGCZ and BTG3-CCDC40 were validated by conventional Sanger sequencing. The genomic locations of all the translocations were labeled in Figure 5.

\section{Analysis of copy number variants}

We identified putative genome-wide copy number variants according to the total read depth and alternative allele proportion at each SNP/INDEL locus. First, we plotted the genome-wide distribution of the alternative allele frequency (also referred to as the B allele frequency or BAF) of the primary and metastatic tumor tissues (Figure 6). We found that the general distribution of the BAF was conserved between the 2 primary and metastatic tumor tissues. The SCNAs were estimated by combining the total read depth and the BAF at each locus. A total of 668 SCNAs in the primary tumor and 553 SCNAs in the metastatic tumor were identified. Next, we focused on the SCNAs that mapped to known genes and were observed in both of the primary and metastatic tumors tissues (Table 3).

\section{Microsatellite instability analysis}

We extracted the genomic locations of the genomic repeats and the changes in the somatic repeat count identified using the SpeedSeq framework. Additional information regarding the repeat regions identified in the primary and lymphatic metastatic GB-SCNEC tissues is provided in Supplementary Table 4 and Table 5 respectively. Repeat motif counts $\geq 4$ and somatic count changes $>5$ were filtered (Table 4). The genomic locations of the repeat motifs with changes in repeat number are also indicated in Figure 3A and Figure 3B. Genome-wide microsatellite instability was observed in both the primary and metastatic GB-SCNECs tissue, which is consistent with observations from many previous studies. 


\section{Discussion}

GB-SCNEC is a relatively rare and aggressive tumor that is associated with a poor prognosis, and early diagnosis with prompt surgical intervention is currently the only treatment that provides favorable long-term outcomes. Therefore, novel insights into the molecular mechanisms underlying GB-SCNEC are required for the development of effective treatment strategies. In the present study, we performed whole-genome sequencing of primary gallbladder cancer, lymphatic metastatic cancer and matched non-cancerous tissues and identified 295 and 712 somatic SNVs in the primary and metastatic tumor tissues, respectively. The genetic aberrations were mapped to genes associated with axon guidance, ERBB signaling, sulfur metabolism and calcium signaling. Among these genes, HMCN1 and CDH10 were determined to be novel suppressors of gallbladder cancer metastasis. In addition, we identified 11 deletions, 4 tandem duplications and 5 inversions that mapped to known genes. Copy number variants were also analyzed, and genome-wide microsatellite instability was observed in both the primary and metastatic tumor specimens.

We previously reported that the ERBB signaling pathway is associated with the development of gallbladder cancer. Using whole-exome and targeted sequencing technology, we identified 12 genes that are involved in the ERBB signaling pathway and associated with somatic mutations in gallbladder cancer, and they included ERBB4, HRAS and NRG1(Li et al. 2014). In the present study, we identified somatic mutations in ERBB4, HRAS and NRG1 in gallbladder cancer tissue. Together, these findings further validate the critical role of the ERBB signaling pathway in gallbladder carcinogenesis.

CDH10 encodes a type II classical cadherin that mediates calcium-dependent cell-cell adhesion. In this study, we identified a somatic mutation in CDH10 with a deleterious effect on the structure and function of certain proteins. The mutation was observed in both the primary and metastatic tumor tissue, indicating that it is associated with tumor metastasis. A relationship between $\mathrm{CDH} 10$ and tumor metastasis has also been observed in colorectal cancer. Jun Yu et al. found that mutations in CDH10 as well as 4 other genes (COL6A3, SMAD4, TMEM132D, VCAN) were significantly associated with improved overall survival in colorectal cancer independent of tumor node metastasis (TNM) stage(Yu et al. 2015). Cadherin-10, the protein encoded by the $\mathrm{CDH} 10$ gene, can directly interact with $\beta$-catenin, a protein that mediates cell-cell interactions(Shimoyama et al. 2000). Notably, cadherins are calcium-dependent cell adhesion proteins, and we identified mutations in 9 genes involved in calcium signaling.

We also identified mutations in 10 genes that play a role in axon guidance signaling; 
therefore, this is the first study to report a link between axon guidance signaling and gallbladder neuroendocrine carcinoma. A relationship between axon guidance signaling and neuroendocrine carcinoma has also been observed in small intestine neuroendocrine tumors (SI-NETs) as reported by Banck MS et al.(Banck et al. 2013). Using exome sequencing, these authors found that mutations in axon guidance were frequently disrupted in SI-NET. In summary, disruptions in axon guidance signaling might play crucial roles in neuroendocrine carcinogenesis.

Structural variations that mapped to known genes were also observed in gallbladder neuroendocrine carcinoma tissues. This study is also the first to report the identification of 11 deletions, 4 tandem duplications and 5 inversions in gallbladder cancer. Notably, a 23,182 bp tandem duplication in chromosome 1 and a 15,645 bp tandem duplication in chromosome 10 were observed in both the primary and metastatic tumor tissues. These duplications might disrupt the expression of genes mapped to these regions (CLCNKA, CLCNKB, FAM131C, C1DP2 and C1DP3), and these potential disruptions in gene expression might play a role in tumor malignancy. NCAM2-SGCZ and BTG3-CCDC40 fusions were among the translocational structure variations. Notably, the functional domains in the 4 genes were partially retained in the fusions, which indicated that novel fusion transcripts were involved in the development and metastasis of gallbladder cancer.

This study is also the first to report the identification of copy number variations in primary and metastatic GB-SCNEC tissues. In addition, microsatellite instability has long been considered a contributor to gallbladder carcinogenesis. The genome-wide microsatellite instability observed in the primary and metastatic gallbladder tumor tissues in this study is consistent with the results from many previous reports(Yoshida et al. 2000; Sessa et al. 2003). In summary, this is first study to report the whole-genome sequencing of matched primary and metastatic gallbladder tumor tissues. Our findings have the potential for use in the identification of novel approaches for predicting and treating lymphatic metastasis in gallbladder cancer.

\section{Reference}

\section{Gallbladder Cancer. American Cancer Society.}

Banck MS, Kanwar R, Kulkarni AA, Boora GK, Metge F, Kipp BR, Zhang L, Thorland EC, Minn KT, Tentu R et al. 2013. The genomic landscape of small intestine neuroendocrine tumors. The Journal of clinical investigation 123(6): 2502-2508.

Belkadi A, Botze A, Itan Y, Cobat A, Vincent QB, Antipenko A, Shang L, Boisson B, 
Casanova JL, Abel L. 2015. Whole-genome sequencing is more powerful than whole-exome sequencing for detecting exome variants. Proceedings of the National Academy of Sciences of the United States of America 112(17): 5473-5478.

Bolger AM, Lohse M, Usadel B. 2014. Trimmomatic: a flexible trimmer for Illumina sequence data. Bioinformatics 30(15): 2114-2120.

Chiang C, Layer RM, Faust GG, Lindberg MR, Rose DB, Garrison EP, Marth GT, Quinlan AR, Hall IM. 2015. SpeedSeq: ultra-fast personal genome analysis and interpretation. Nature methods 12(10): 966-968.

Cibulskis K, Lawrence MS, Carter SL, Sivachenko A, Jaffe D, Sougnez C, Gabriel S, Meyerson M, Lander ES, Getz G. 2013. Sensitive detection of somatic point mutations in impure and heterogeneous cancer samples. Nature biotechnology 31(3): 213-219.

El Fattach H, Guerrache Y, Eveno C, Pocard M, Kaci R, Shaar-Chneker C, Dautry R, Boudiaf M, Dohan A, Soyer P. 2015. Primary neuroendocrine tumors of the gallbladder: Ultrasonographic and MDCT features with pathologic correlation. Diagnostic and interventional imaging 96(5): 499-502.

Faust GG, Hall IM. 2014. SAMBLASTER: fast duplicate marking and structural variant read extraction. Bioinformatics 30(17): 2503-2505.

Henson DE, Albores-Saavedra J, Corle D. 1992. Carcinoma of the gallbladder. Histologic types, stage of disease, grade, and survival rates. Cancer 70(6): 1493-1497.

Itoi T, Watanabe H, Yoshida M, Ajioka Y, Nishikura K, Saito T. 1997. Correlation of p53 protein expression with gene mutation in gall-bladder carcinomas. Pathology international 47(8): 525-530.

Jun SR, Lee JM, Han JK, Choi BI. 2006. High-grade neuroendocrine carcinomas of the gallbladder and bile duct Report of four cases with pathological correlation. Journal of computer assisted tomography 30(4): 604-609.

Lai CH, Lau WY. 2008. Gallbladder cancer--a comprehensive review. The surgeon : journal of the Royal Colleges of Surgeons of Edinburgh and Ireland 6(2): 101-110.

Layer RM, Chiang C, Quinlan AR, Hall IM. 2014. LUMPY: a probabilistic framework for structural variant discovery. Genome biology 15(6): R84.

Li M, Zhang Z, Li X, Ye J, Wu X, Tan Z, Liu C, Shen B, Wang XA, Wu W et al 2014. Whole-exome and targeted gene sequencing of gallbladder carcinoma identifies 
recurrent mutations in the ErbB pathway. Nature genetics 46(8): 872-876.

McKenna A, Hanna M, Banks E, Sivachenko A, Cibulskis K, Kernytsky A, Garimella K, Altshuler D, Gabriel S, Daly M et al. 2010. The Genome Analysis Toolkit: a MapReduce framework for analyzing next-generation DNA sequencing data. Genome research 20(9): 1297-1303.

McLaren W, Pritchard B, Rios D, Chen Y, Flicek P, Cunningham F. 2010. Deriving the consequences of genomic variants with the Ensembl API and SNP Effect Predictor. Bioinformatics 26(16): 2069-2070.

Miyahara N, Shoda J, Kawamoto T, Ishida H, Ueda T, Akimoto Y, Kawakami H, Irimura T. 2014. Interaction of Muc4 and ErbB2 in a transgenic mouse model of gallbladder carcinoma: potential pathobiological implications. Oncology reports 32(5): 1796-1802.

Saetta AA. 2006. K-ras, p53 mutations, and microsatellite instability (MSI) in gallbladder cancer. Journal of surgical oncology 93(8): 644-649.

Sessa F, Furlan D, Genasetti A, Billo P, Feltri M, Capella C. 2003. Microsatellite instability and p53 expression in gallbladder carcinomas. Diagnostic molecular pathology: the American journal of surgical pathology, part B 12(2): 96-102.

Shimoyama Y, Tsujimoto G, Kitajima M, Natori M. 2000. Identification of three human type-II classic cadherins and frequent heterophilic interactions between different subclasses of type-II classic cadherins. The Biochemical journal 349(Pt 1): 159-167.

Shugay M, Ortiz de Mendibil I, Vizmanos JL, Novo FJ. 2013. Oncofuse: a computational framework for the prediction of the oncogenic potential of gene fusions. Bioinformatics 29(20): 2539-2546.

Yao JC, Hassan M, Phan A, Dagohoy C, Leary C, Mares JE, Abdalla EK, Fleming JB, Vauthey JN, Rashid A et al. 2008. One hundred years after "carcinoid": epidemiology of and prognostic factors for neuroendocrine tumors in 35,825 cases in the United States. Journal of clinical oncology : official journal of the American Society of Clinical Oncology 26(18): 3063-3072.

Yoshida T, Sugai T, Habano W, Nakamura S, Uesugi N, Funato O, Saito K. 2000. Microsatellite instability in gallbladder carcinoma: two independent genetic pathways of gallbladder carcinogenesis. Journal of gastroenterology 35(10): 768-774. 
Yu J, Wu WK, Li X, He J, Li XX, Ng SS, Yu C, Gao Z, Yang J, Li M et al. 2015. Novel recurrently mutated genes and a prognostic mutation signature in colorectal cancer. Gut 64(4): 636-645.

Zhang Z, Hao K. 2015. SAAS-CNV: A Joint Segmentation Approach on Aggregated and Allele Specific Signals for the Identification of Somatic Copy Number Alterations with Next-Generation Sequencing Data. PLoS computational biology 11(11): e1004618.

Zhu AX, Hong TS, Hezel AF, Kooby DA. 2010. Current management of gallbladder carcinoma. The oncologist 15(2): 168-181.

\section{Acknowledgments}

This study was supported by the National Natural Science Foundation of China (No. 81172026, 81272402, 81301816, 81172029, 91440203, 81402403, 81502433 and 31501127), the National High Technology Research and Development Program (863 Program) (No. 2012AA022606), the Foundation for Interdisciplinary Research of Shanghai Jiao Tong University (No. YG2011ZD07), the Shanghai Science and Technology Commission Intergovernmental International Cooperation Project (No. 12410705900), the Shanghai Science and Technology Commission Medical Guiding Project (No. 12401905800), the Program for Changjiang Scholars, the Natural Science Research Foundation of Shanghai Jiao Tong University School of Medicine (No. 13XJ10037), the Leading Talent program of Shanghai and Specialized Research Foundation for the PhD Program of Higher Education-Priority Development Field (No. 20130073130014), the Interdisciplinary Program of Shanghai Jiao Tong University (No.14JCRY05), the Shanghai Rising-Star Program (No. 15QA1403100), Top 6 elitist summit of jiangsu Province (No. 2013-WSN-025), and the People's Livelihood Science and Technology Demonstration Project of Yixing City (No. 2014-80). 


\section{Figure Legends}

Figure 1. Clinical data of the patient with GB-SCNEC. (A, B) MDCT of the abdomen in the axial plane after the intravenous administration of iodinated contrast material revealed a heterogeneous mass (black arrow) in the neck of the gallbladder in association with large heterogeneous lymphadenopathies (red arrow) in the porta hepatis space. (C, D) Microscopic features of primary gallbladder cancer (C) and metastatic lymph node tissues (D) observed using hematoxylin and eosin (H\&E) staining. (E-L) Immunohistochemistry analysis revealed that the tumor cells were negative for chromogranin A but positive (red arrow) for synaptophysin, NSE and Ki67 (80\% of cells) in the primary gallbladder cancer (E-H) and metastatic lymph node tissues (I-L).

Figure 2. Genomic distribution of SNVs detected in the primary and metastatic GB-SCNEC tissues. In total, 295 and 712 somatic SNVs in primary and lymphatic metastatic tumors were identified, respectively, and 109 somatic SNVs were observed in both the primary and metastatic tumor tissues. The majority of the SNVs were located in intronic and intergenic regions.

Figure 3. Chromosomal distribution of SNVs, structural variations, copy number variations and microsatellite instability identified in the primary and metastatic GB-SCNECs. (A) Chromosomal distributions of SNVs (red), structural variations (green), copy number variations (yellow) and microsatellite instabilities (purple) observed in the primary GB-SCNEC. (B) Chromosomal distribution of the corresponding variations in the metastatic GB-SCNEC. 
Figure 4. Pathway analysis of the genes with somatic SNVs, functional predictions and sanger sequencing validations of SNVs in HMCN1 and CDH10. (A) Pathway analysis of the somatic genes mapped to SNVs. The genes associated with axon guidance, ERBB signaling, sulfur metabolism and calcium signaling were significantly enriched. (B) Amino acid changes in the proteins encoded by HMCN1 (E171K) and CDH10 (R4623W) had a damaging effect on the structure and function of proteins. (C) The SNVs associated with the amino acid changes were validated using conventional Sanger sequencing.

Figure 5. Genomic locations of all the translocational structure variants discovered in the primary and metastatic tumors. The translocatianal regions were linked by lines with different colors (black lines for those specially identified in one of the two tumors, blue lines for those identified in both of the tumors and reds lined for gene fusions).

Figure 6. Genome-wide distribution of the alternative allele proportion (also referred to as the B allele frequency, BAF) in the primary and metastatic tumor tissue. 

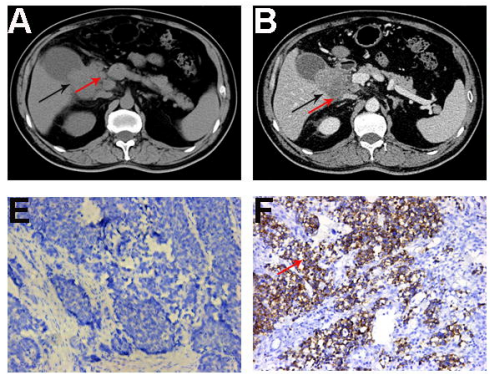

I

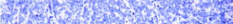

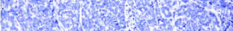

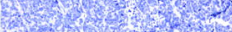

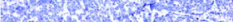

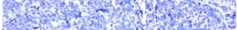

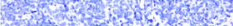
30 to
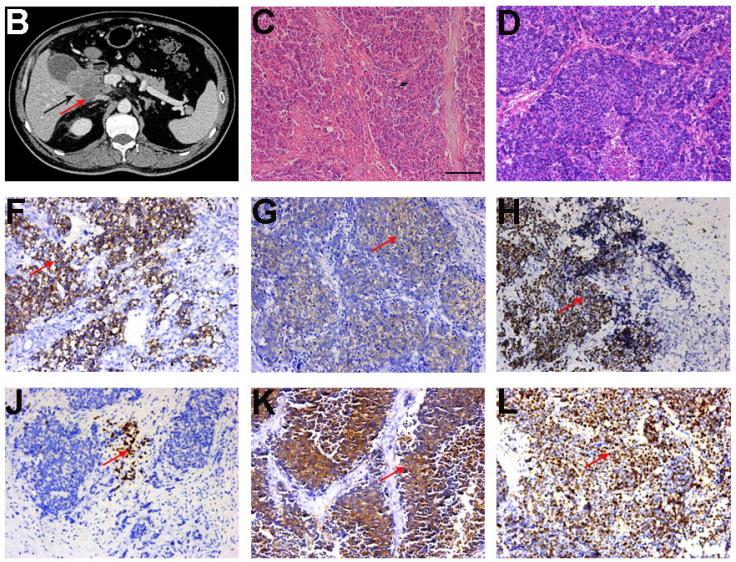


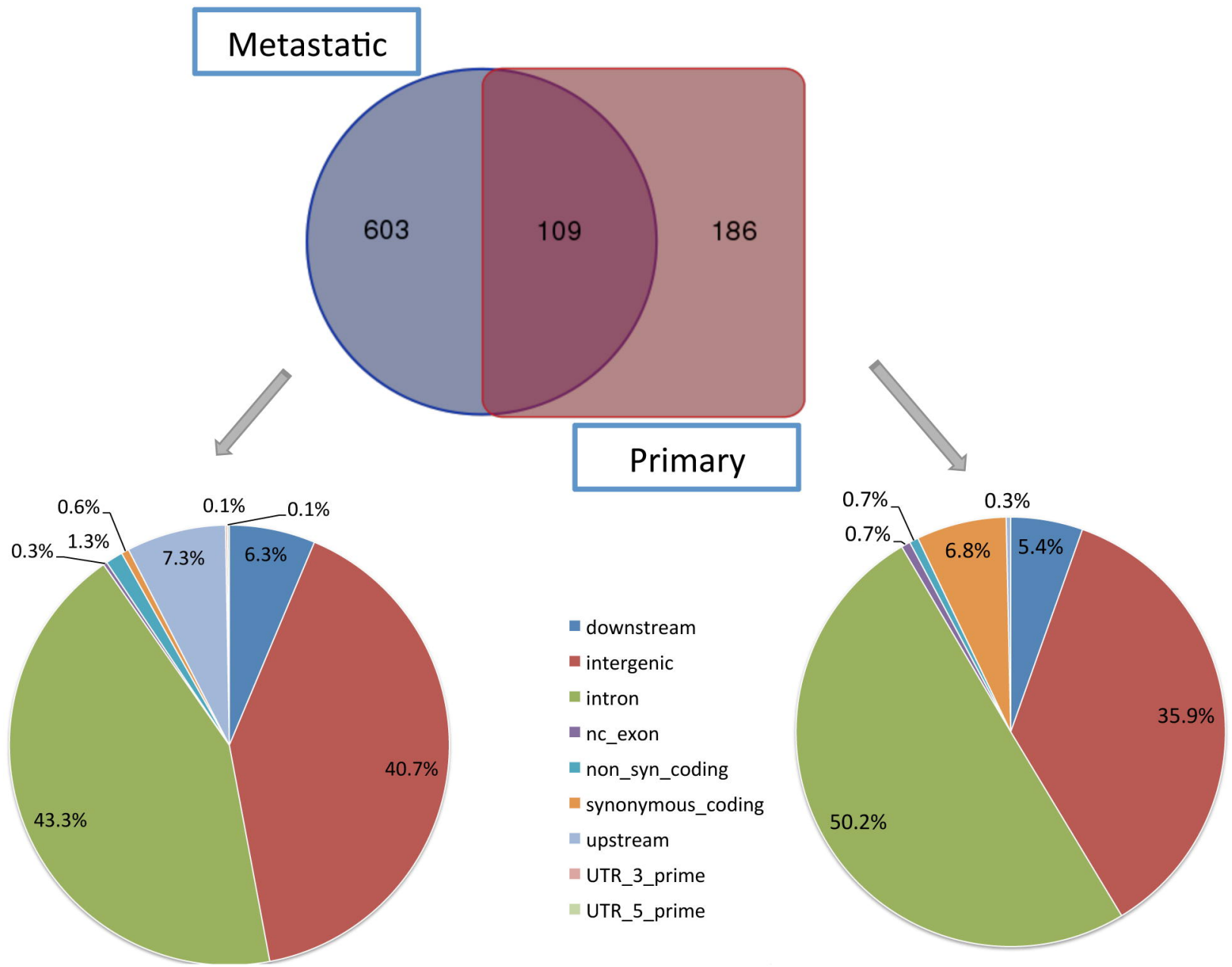




\section{Primary tumor}

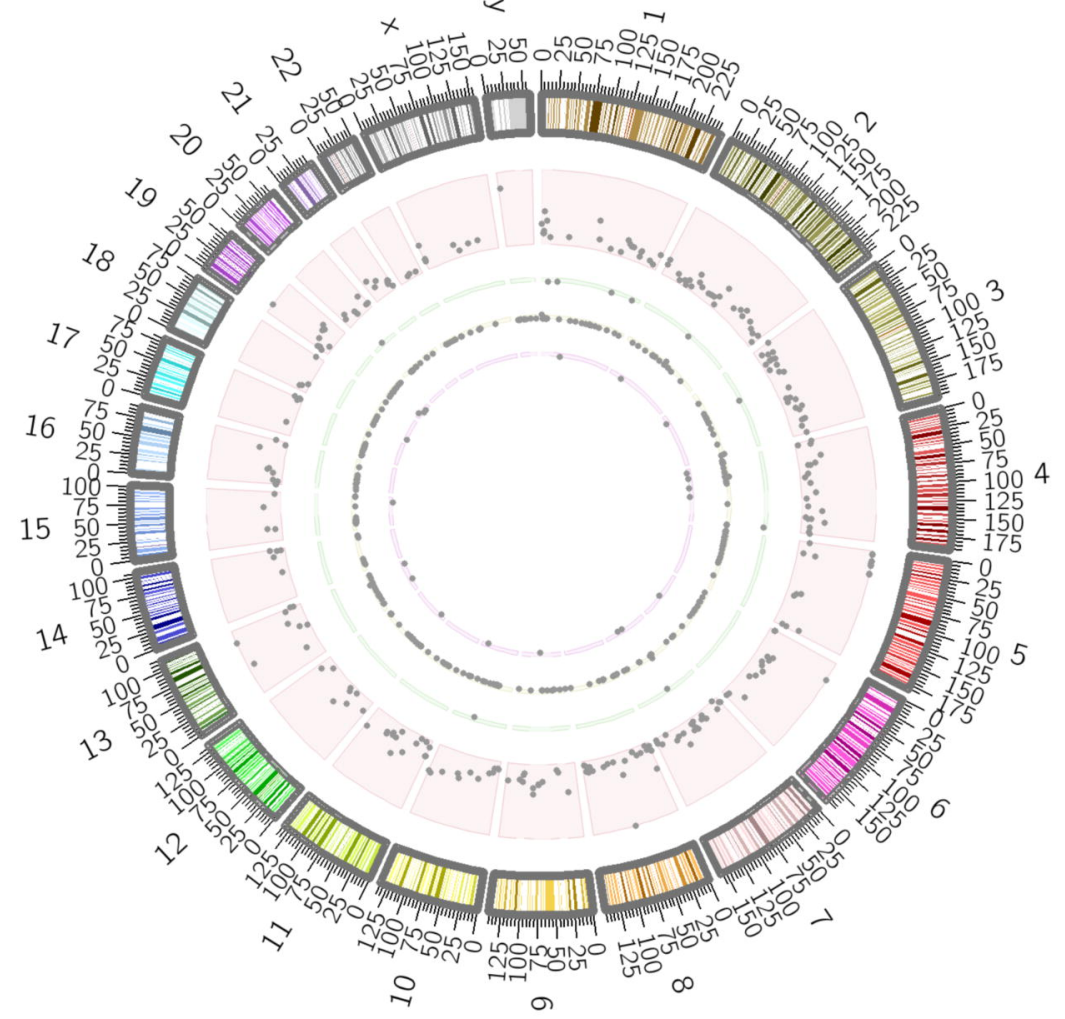

\section{Metastatic tumor}

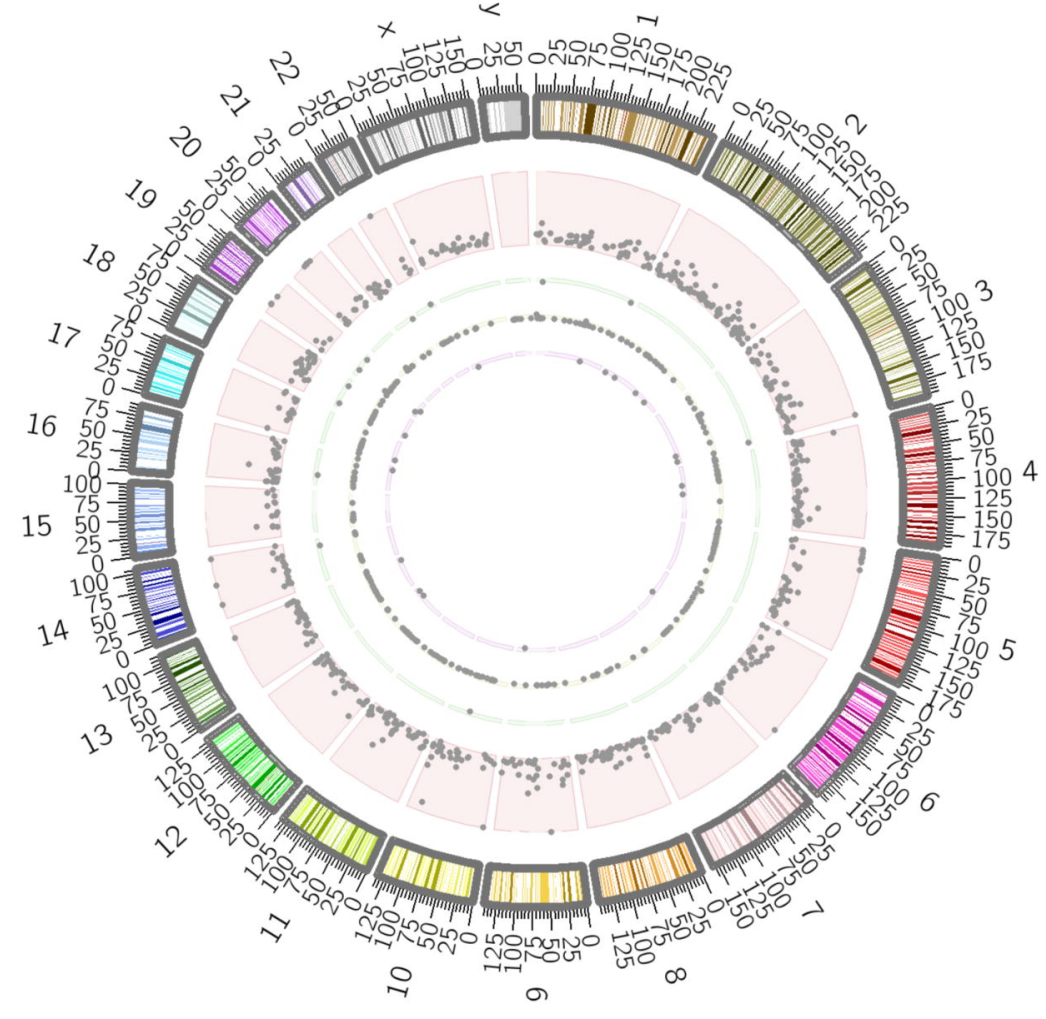


bioRxiv preprint doi: https://doi.org/10.1101/052316; this version posted May 9, 2016. The copyright holder for this preprint (which was not certified by peer review) is the author/funder, who has granted bioRxiv a license to display the preprint in perpetuity. It is made available under
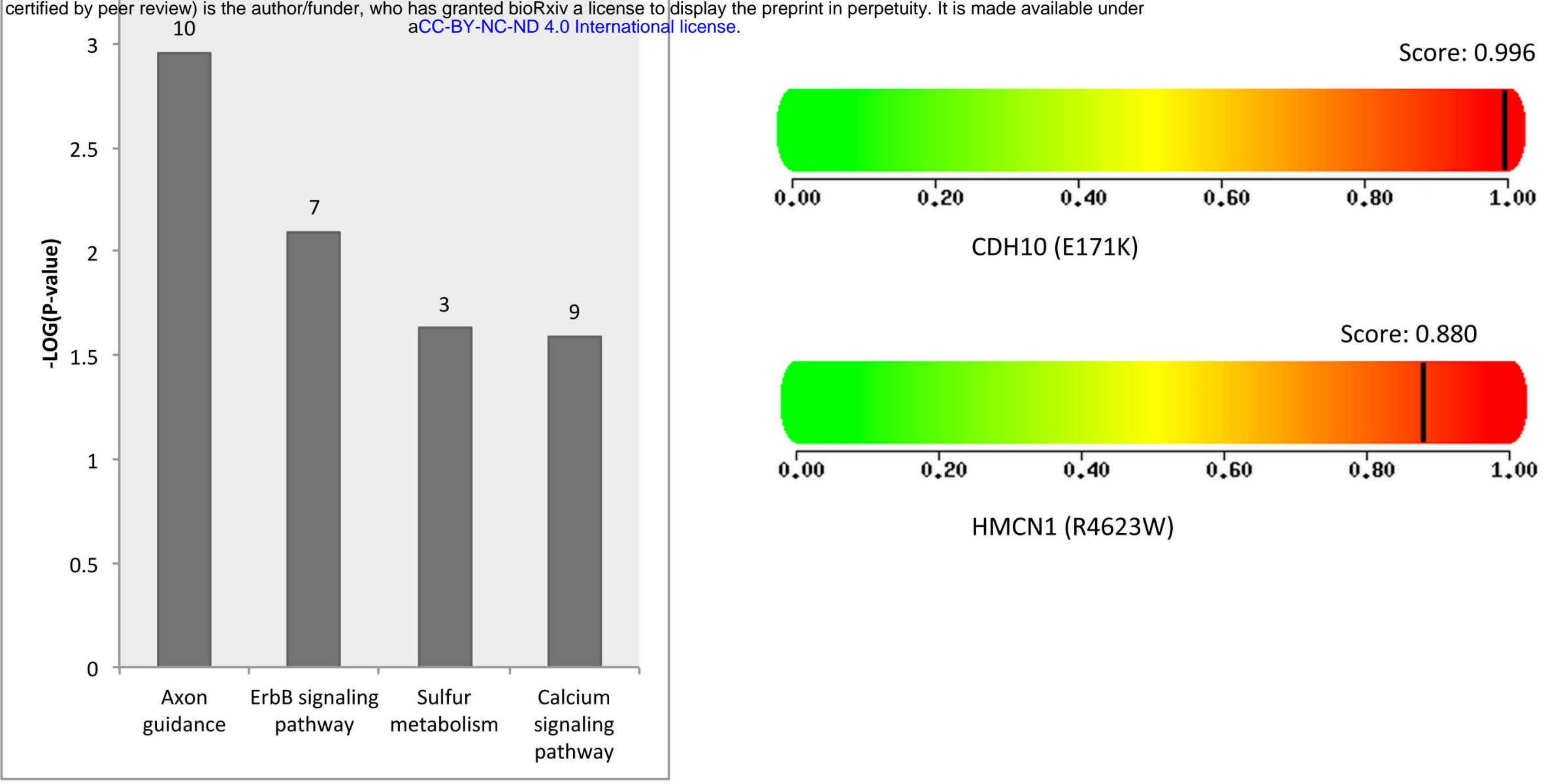

\section{C}
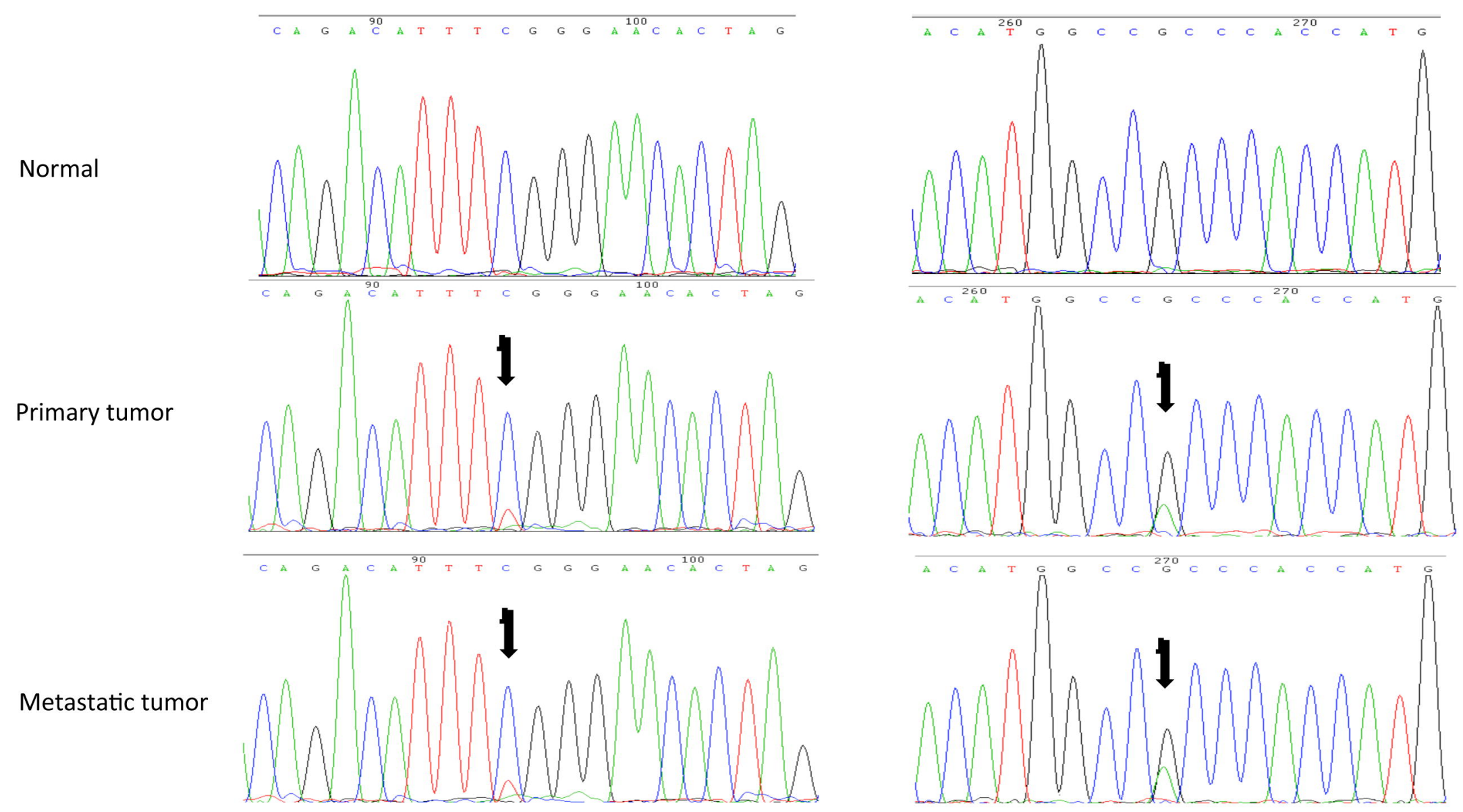

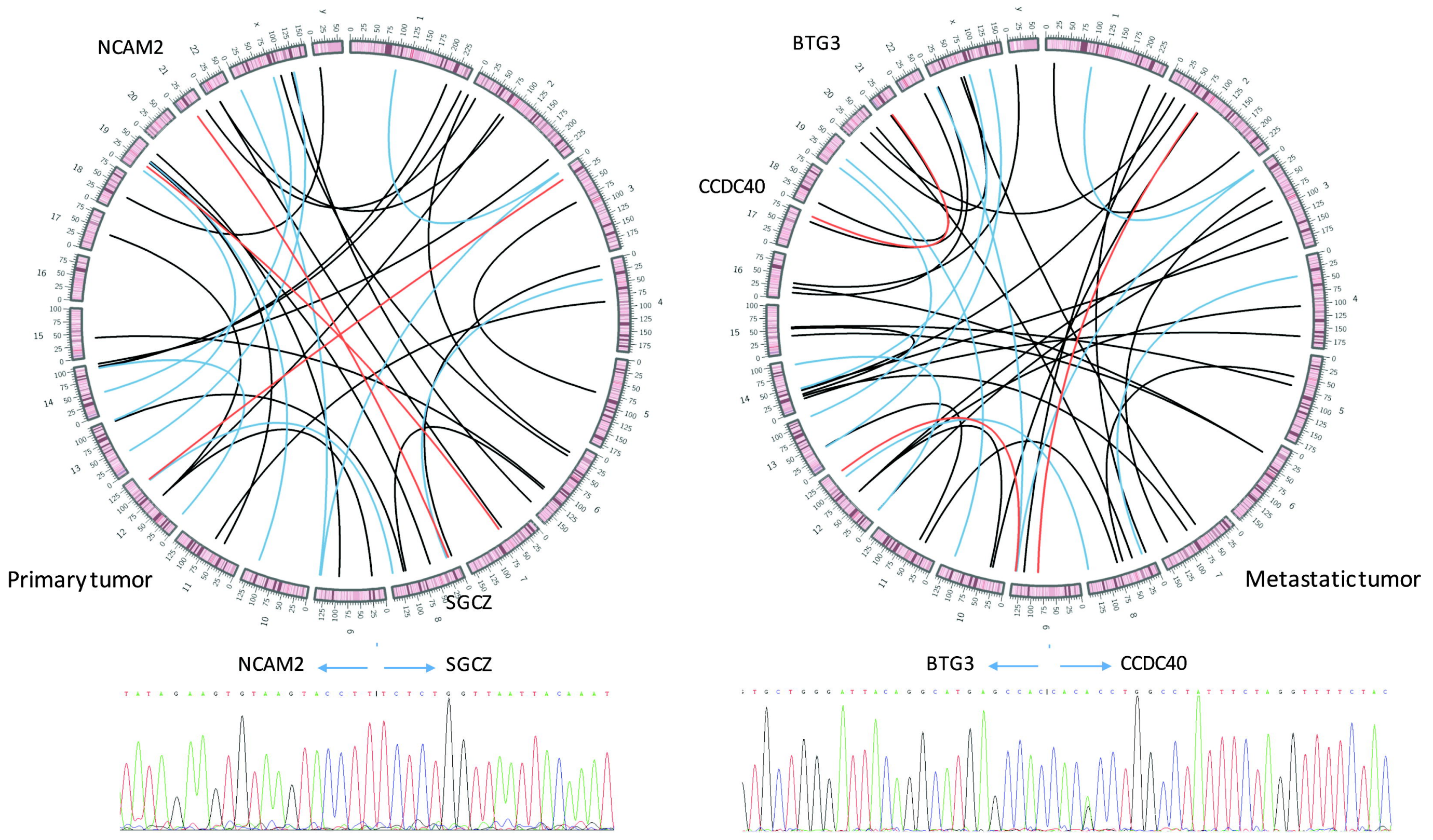

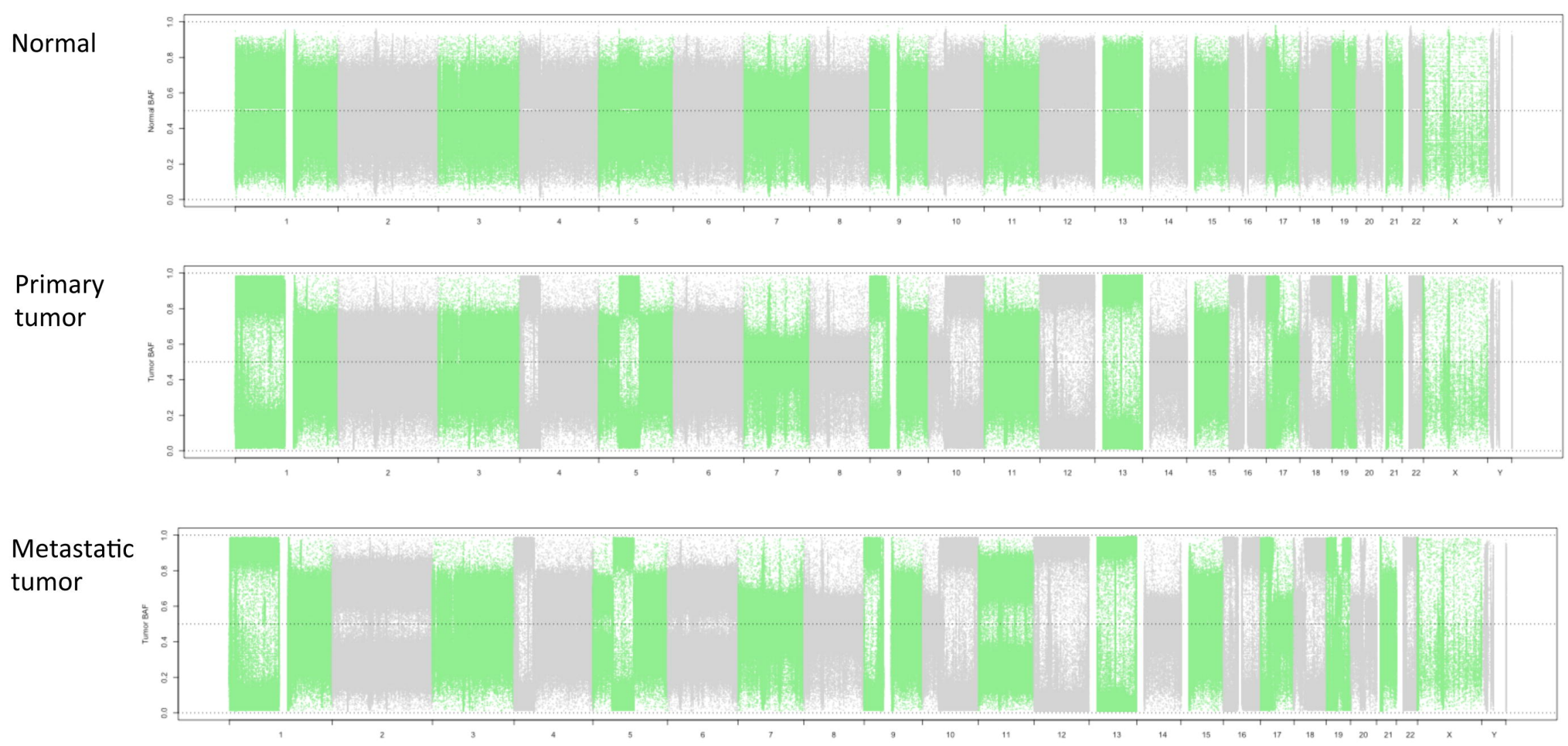

Chromosomes 
Table1. Somatic SNVs discovered by whole genome sequencing

\begin{tabular}{|c|c|c|c|c|c|c|c|c|}
\hline Gene & Chr. & Position & Allele change & $\begin{array}{l}\text { Amino acid } \\
\text { change }\end{array}$ & Gerp score & Cadd score & Impact & Tumors observed \\
\hline HMCN1 & 1 & 186107047 & $\mathrm{C}>\mathrm{T}$ & $\mathrm{R}>\mathrm{W}$ & 3.47 & 3.29 & non_syn_coding & Both \\
\hline MAST2 & 1 & 46487740 & $\mathrm{G}>\mathrm{A}$ & $\mathrm{R}>\mathrm{Q}$ & 5.74 & 6.00 & non_syn_coding & Metastatic \\
\hline CDH10 & 5 & 24537504 & $\mathrm{C}>\mathrm{T}$ & $E>K$ & 5.72 & 3.72 & non_syn_coding & Both \\
\hline C6orf118 & 6 & 165715716 & $\mathrm{G}>\mathrm{A}$ & $\mathrm{T}>\mathrm{M}$ & 0.45 & -0.10 & non_syn_coding & Metastatic \\
\hline UBR5 & 8 & 103306037 & $\mathrm{C}>\mathrm{T}$ & $\mathrm{R}>\mathrm{H}$ & 6.05 & 4.06 & non_syn_coding & Metastatic \\
\hline OR51F1 & 11 & 4790457 & $\mathrm{C}>\mathrm{A}$ & $A>S$ & 3.33 & 1.23 & non_syn_coding & Metastatic \\
\hline SLC22A8 & 11 & 62782405 & $\mathrm{C}>\mathrm{T}$ & $\mathrm{R}>\mathrm{H}$ & 3.85 & 2.76 & non_syn_coding & Metastatic \\
\hline CSPG4 & 15 & 75982085 & $\mathrm{C}>\mathrm{T}$ & $E>K$ & 5.26 & 3.58 & non_syn_coding & Metastatic \\
\hline BAIAP2L2 & 22 & 38494168 & $\mathrm{C}>\mathrm{T}$ & $E>K$ & 5.53 & 5.10 & non_syn_coding & Metastatic \\
\hline
\end{tabular}


Table2. Gene related structure variations discovered by whole genome sequencing

\begin{tabular}{|c|c|c|c|c|c|c|}
\hline Type & $\begin{array}{c}\text { Chromo } \\
\text { some }\end{array}$ & Breakpoint A & Breakpoint B & Length (bp) & Tumors observed & Associated genes \\
\hline Deletion & 1 & 246188189 & 246188673 & 484 & Metastatic & SMYD3 \\
\hline Deletion & 1 & 230470943 & 230471300 & 357 & Primary & PGBD5 \\
\hline Deletion & 3 & 193016236 & 193017447 & 1211 & Metastatic & ATP13A5 \\
\hline Deletion & 4 & 187093486 & 187098241 & 4755 & Primary & FAM149A \\
\hline Deletion & 14 & 88309673 & 88310680 & 1007 & Metastatic & GALC \\
\hline Deletion & 16 & 25062127 & 25096342 & 34215 & Metastatic & SCML2P2 \\
\hline Deletion & 18 & 61312483 & 61330089 & 17606 & Metastatic & SERPINB3 \\
\hline Deletion & 19 & 47286528 & 47326398 & 39870 & Metastatic & HNRNPMP2, SLC1A5 \\
\hline Deletion & 20 & 25449141 & 25449972 & 831 & Primary & NINL \\
\hline Deletion & 21 & 47803700 & 47804099 & 399 & Metastatic & PCNT \\
\hline Deletion & 22 & 42524892 & 42538705 & 13813 & Metastatic & CYP2D7P, CYP2D6 \\
\hline Tandem duplication & 1 & 16363336 & 16386518 & 23182 & Both & CLCNKA, CLCNKB, FAM131C \\
\hline Tandem duplication & 3 & 77340220 & 77344873 & 4653 & Primary & $\mathrm{ROBO} 2$ \\
\hline Tandem duplication & 10 & 81789166 & 81804811 & 15645 & Both & $\mathrm{C} 1 \mathrm{DP} 2, \mathrm{C} 1 \mathrm{DP} 3$ \\
\hline Tandem duplication & 18 & 11611133 & 11645266 & 34133 & Metastatic & NPIPB1P \\
\hline Inversion & 1 & 39958851 & 40252955 & 294104 & Primary & BMP8A \\
\hline Inversion & 1 & 175965396 & 175973594 & 8198 & Primary & RFWD2 \\
\hline Inversion & 2 & 130810429 & 132042135 & 1231706 & Primary & CYP4F27P \\
\hline Inversion & 7 & 76253268 & 76580290 & 327022 & Primary & UPK3B, POMZP3 \\
\hline Inversion & 19 & 16171830 & 16181526 & 9696 & Metastatic & CTD-2231E14.4 \\
\hline
\end{tabular}


Table3. Copy number variations discovered by whole genome sequencing

\begin{tabular}{|c|c|c|c|c|c|c|c|}
\hline Chr & Start & End & Length & Locus number & CNV type & Gene number & Gene name \\
\hline $\operatorname{chr} 4$ & 3479562 & 3636112 & 156551 & 1912 & loss & 3 & DOK7 et al. \\
\hline $\operatorname{chr} 4$ & 34037877 & 45014591 & 10976715 & 8635 & loss & 51 & APBB2 et al. \\
\hline chr5 & 15890 & 3323754 & 3307865 & 4788 & gain & 35 & AHRR et al. \\
\hline chr5 & 21479448 & 21480563 & 1116 & 12 & loss & 1 & GUSBP1 \\
\hline chr5 & 34195054 & 45953915 & 11758862 & 7994 & gain & 66 & AGXT2 et al. \\
\hline $\operatorname{chr} 10$ & 49745229 & 51195765 & 1450537 & 894 & loss & 18 & ARHGAP22 et al. \\
\hline $\operatorname{chr} 17$ & 171973 & 1755819 & 1583847 & 2072 & loss & 34 & ABR et al. \\
\hline $\operatorname{chr} 17$ & 20786423 & 21193432 & 407010 & 438 & loss & 6 & C17orf103 et al. \\
\hline $\operatorname{chr} 17$ & 30144873 & 30285495 & 140623 & 51 & loss & 3 & COPRS et al. \\
\hline $\operatorname{chr} 18$ & 106728 & 112535 & 5808 & 351 & loss & 1 & ROCK1P1 \\
\hline $\operatorname{chr} 18$ & 125075 & 805150 & 680076 & 840 & loss & 9 & C18orf56 et al. \\
\hline $\operatorname{chr} 18$ & 23627315 & 24376447 & 749133 & 139 & loss & 5 & KCTD1 et al. \\
\hline chr19 & 8747389 & 9028188 & 280800 & 644 & loss & 5 & ACTL9 et al. \\
\hline chr19 & 17044763 & 20685187 & 3640425 & 2640 & loss & 104 & ABHD8 et al. \\
\hline chr19 & 22618698 & 23481974 & 863277 & 1333 & loss & 6 & LOC440518 et al. \\
\hline chr19 & 34885372 & 36747800 & 1862429 & 1653 & gain & 77 & ALKBH6 et al. \\
\hline $\operatorname{chr} 20$ & 25734683 & 25764502 & 29820 & 662 & loss & 1 & FAM182B \\
\hline $\operatorname{chr} 22$ & 17278454 & 17338017 & 59564 & 71 & loss & 2 & HSFY1P1 et al. \\
\hline $\operatorname{chr} 22$ & 20716839 & 21461982 & 745144 & 964 & loss & 20 & AIFM3 et al. \\
\hline $\operatorname{chr} 22$ & 22002021 & 22578991 & 576971 & 930 & loss & 7 & MAPK1 et al. \\
\hline $\operatorname{chr} Y$ & 2759925 & 9950526 & 7190602 & 2424 & loss & 35 & AMELY et al. \\
\hline
\end{tabular}


Table 4 Microsatellite instability regions discovered by whole genome sequencing

\begin{tabular}{|c|c|c|c|c|c|c|}
\hline Chr. & Location & Motif & Length in normal & $\begin{array}{l}\text { Length in } \\
\text { tumor }\end{array}$ & Repeat count change & Tumors observed \\
\hline chr1 & 69703144 & TAGA & 38 & 66 & 7 & Primary \\
\hline chr1 & 167530083 & GAAA & 91 & 443 & 88 & Metastatic \\
\hline chr2 & 5990493 & TTTA & 74 & 494 & 105 & Metastatic \\
\hline chr2 & 45531195 & TTTC & 45 & 77 & 8 & Both \\
\hline chr2 & 191250792 & TTTTA & 94 & 49 & -9 & Metastatic \\
\hline chr2 & 144313429 & TAGAA & 67 & 27 & -8 & Metastatic \\
\hline chr3 & 28079626 & TTTC & 94 & 362 & 67 & Primary \\
\hline chr3 & 192285524 & TTTC & 123 & 359 & 59 & Metastatic \\
\hline chr3 & 170118525 & TTTC & 124 & 548 & 106 & Metastatic \\
\hline $\mathrm{chr} 4$ & 71206459 & TTTA & 19 & 51 & 8 & Both \\
\hline chr4 & 36333809 & TAAAA & 84 & 54 & -6 & Primary \\
\hline chr4 & 13712785 & TAAA & 39 & 67 & 7 & Primary \\
\hline chr4 & 103546995 & TTTTA & 64 & 34 & -6 & Both \\
\hline chr6 & 91122413 & GGAAA & 38 & 68 & 6 & Both \\
\hline chr7 & 92678135 & CAAAA & 54 & 114 & 12 & Primary \\
\hline chr7 & 108898557 & TTTC & 66 & 230 & 41 & Primary \\
\hline chr9 & 74371107 & TAGA & 22 & 46 & 6 & Primary \\
\hline chr9 & 112301512 & TTTC & 113 & 385 & 68 & Metastatic \\
\hline $\operatorname{chr} 10$ & 115017138 & TTTC & 123 & 603 & 120 & Primary \\
\hline chr12 & 94909841 & AGAAA & 43 & 88 & 9 & Metastatic \\
\hline chr12 & 19265351 & GAAAA & 71 & 41 & -6 & Primary \\
\hline chr12 & 119158255 & TTTC & 141 & 285 & 36 & Metastatic \\
\hline chr13 & 42832403 & TTTA & 37 & 61 & 6 & Primary \\
\hline
\end{tabular}




\begin{tabular}{|c|c|c|c|c|c|c|}
\hline chr13 & 104355487 & TTTC & 101 & 73 & -7 & Both \\
\hline chr15 & 84001991 & TTTC & 113 & 577 & 116 & Primary \\
\hline chr16 & 77801681 & GAAA & 51 & 199 & 37 & Metastatic \\
\hline chr17 & 9672167 & TTTA & 36 & 60 & 6 & Metastatic \\
\hline chr18 & 2158902 & GAAA & 75 & 239 & 41 & Primary \\
\hline chr18 & 12298904 & GGGA & 61 & 321 & 65 & Metastatic \\
\hline chr19 & 40429543 & GAAA & 44 & 192 & 37 & Primary \\
\hline chr19 & 31435307 & TAAA & 51 & 107 & 14 & Primary \\
\hline chr19 & 28323640 & TTTTA & 106 & 341 & 47 & Metastatic \\
\hline chr19 & 15971928 & TTTC & 55 & 519 & 116 & Both \\
\hline chrX & 22515660 & TCTA & 62 & 2 & -15 & Metastatic \\
\hline
\end{tabular}

